\title{
Independent Vesicle Pools Underlie Different Modes of Release during Neuronal Development
}

\author{
Laura C. Andreae, Naila Ben Fredj, and Juan Burrone \\ Medical Research Council Centre for Developmental Neurobiology, Kings College London, London SE1 1UL, United Kingdom
}

Mature presynaptic terminals release neurotransmitter both in response to activity and spontaneously. We found that axons of rat hippocampal neurons initially show very high levels of exclusively spontaneous release, which progressively switches over to the mature phenotype during synapse formation. These two modes of vesicle cycling derive from distinct pools throughout development and the initiation of activity-dependent release was independent of postsynaptic contacts, suggesting it is an autonomous presynaptic event.

\section{Introduction}

At mature synapses, information is transferred by the fusion of synaptic vesicles with the plasma membrane, allowing release of neurotransmitter and subsequent activation of postsynaptic receptors. Vesicle fusion occurs both in response to depolarization of the presynaptic membrane, following the arrival of an action potential, and spontaneously. Recent work has described the identity of the pools of vesicles responsible for each form of release, although debate has surrounded the issue of whether spontaneous vesicular release at mature synapses derives from the same pool of vesicles as evoked release (Groemer and Klingauf, 2007; Hua et al., 2010; Wilhelm et al., 2010) or constitutes a separate pool that is mobilized independently (Sara et al., 2005; Atasoy et al., 2008; Fredj and Burrone, 2009; Chung et al., 2010).

During neuronal development, a progressive increase in the number of vesicles at the presynapse during development has been described (Blue and Parnavelas, 1983; Mozhayeva et al., 2002). While studies in peripheral and central neuronal culture systems have shown that synapses appear to be capable of both spontaneous and activity-dependent vesicle cycling early in development and possibly before axodendritic contact (Matteoli et al., 1992; Kraszewski et al., 1995; Dai and Peng, 1996; Zakharenko et al., 1999; Sabo and McAllister, 2003; Tojima et al., 2007), there has been no careful examination of the identity of vesicle pools and their mode of mobilization. Previous studies have characterized the functional rearrangements that occur in the pool of vesicles responsible for evoked release during development (Mozhayeva et al., 2002), but do not establish how this relates to spontaneous release nor how contact with the postsynaptic compartment may influence maturation. Finally, nothing is known about the origins of the evoked and spontaneous pools of vesicles during development.

Received 0ct. 14, 2011; revised Nov. 18, 2011; accepted Dec. 12, 2011.

Author contributions: L.C.A., N.B.F., and J.B. designed research; L.C.A. and N.B.F. performed research; L.C.A., N.B.F., and J.B. analyzed data; L.C.A. and J.B. wrote the paper.

This work was supported by a Medical Research Council project grant and a Lister prize fellowship to J.B.

Correspondence should be addressed to Juan Burrone, Medical Research Council Centre for Developmental Neu-

robiology, Kings College London, New Hunts House, Guys Hospital Campus, London SE1 1UL, UK. E-mail: juan.burrone@kcl.ac.uk.

DOI:10.1523/JNEUROSCI.5181-11.2012

Copyright $\odot 2012$ the authors $\quad 0270-6474 / 12 / 321867-08 \$ 15.00 / 0$
We therefore performed a detailed study of activity-dependent and spontaneous vesicle cycling in dissociated hippocampal neurons during development. We found that the mode of release switches from exclusively spontaneous early on in development to predominantly evoked in mature neurons. This switch in the release mode was independent of postsynaptic contacts, suggesting it is a cell-autonomous presynaptic phenomenon. Importantly, separate pools underlie the two forms of release throughout development and at maturity, further strengthening the notion that spontaneous and evoked release use independent vesicle pools.

\section{Materials and Methods}

Hippocampal cultures. Primary hippocampal cultures were prepared from embryonic day 18 Sprague Dawley rats of either sex. Hippocampi were dissociated using trypsin $\left(5 \mathrm{mg} / \mathrm{ml}\right.$ for $15 \mathrm{~min}$ at $37^{\circ} \mathrm{C}$; Worthington $)$ and plated at 350 cells $/ \mathrm{mm}^{2}$ on glass coverslips coated with poly-D-lysine $(50 \mu \mathrm{g} / \mathrm{ml})$ and laminin $(20 \mu \mathrm{g} / \mathrm{ml})$. Neurons were incubated $\left(37^{\circ} \mathrm{C}, 6 \% \mathrm{CO}_{2}\right)$ in neurobasal media supplemented with B27 (2\%) and glutamax (500 $\mu \mathrm{M})$. After 3-6 din culture, neurons were transfected using Lipofectamine 2000. Unless otherwise specified, all reagents were from Invitrogen.

Labeling vesicles with biosyn and immunocytochemistry. Primary hippocampal neurons were cotransfected with VAMP2-BAP and BirA plasmids (Fredj and Burrone, 2009) at 3-6 din vitro (DIV) and $100 \mu \mathrm{m}$ biotin added. Vesicles were labeled as previously described (Fredj and Burrone, 2009). Briefly, experiments were performed in HEPES-buffered saline (HBS) containing the following (in mM): $139 \mathrm{NaCl}, 2.5 \mathrm{KCl}, 10$ HEPES, 10 D-glucose, $2 \mathrm{CaCl}_{2}, 1.3 \mathrm{MgCl}_{2}, 1 \mu \mathrm{M}$ TTX (Sigma), $25 \mu \mathrm{M}$ AP5 (Sigma), and $20 \mu \mathrm{M}$ CNQX (pH 7.3, $290 \mathrm{mOsmol}$; Sigma). Neurons were incubated for $30 \mathrm{~s}$ in either streptavidin-Alexa 488 (1:250) or unlabeled streptavidin to label surface VAMP2. To label activity-dependent vesicles, neurons were depolarized for $90 \mathrm{~s}$ in HBS containing the following (in $\mathrm{mm}$ ): $78.5 \mathrm{NaCl}, 60 \mathrm{KCl}$ (adjusted to $290 \mathrm{mOsmol}$ ), streptavidinAlexa 555 (1:250). To label vesicles cycling spontaneously, neurons were incubated for $15 \mathrm{~min}$ at $37^{\circ} \mathrm{C}$ in calcium-free HBS plus streptavidinAlexa 555/647. Cells were fixed in 4\% PFA/0.33 m sucrose. For antibody staining, neurons were permeabilized with $0.1 \%$ saponin for $10 \mathrm{~min}$, blocked with $3 \%$ bovine serum albumin (BSA) for $1 \mathrm{~h}$, and incubated in PSD-95 antibody (1:250, 1 h, MA1-045; Pierce) followed by Alexa-647 secondary antibody (1:500, $30 \mathrm{~min})$. Images were obtained using a FV1000 Olympus confocal microscope.

Synaptophysin-pHluorin imaging. Experiments were done as previously described in neurons expressing synaptophysin-pHluorin (Fredj 


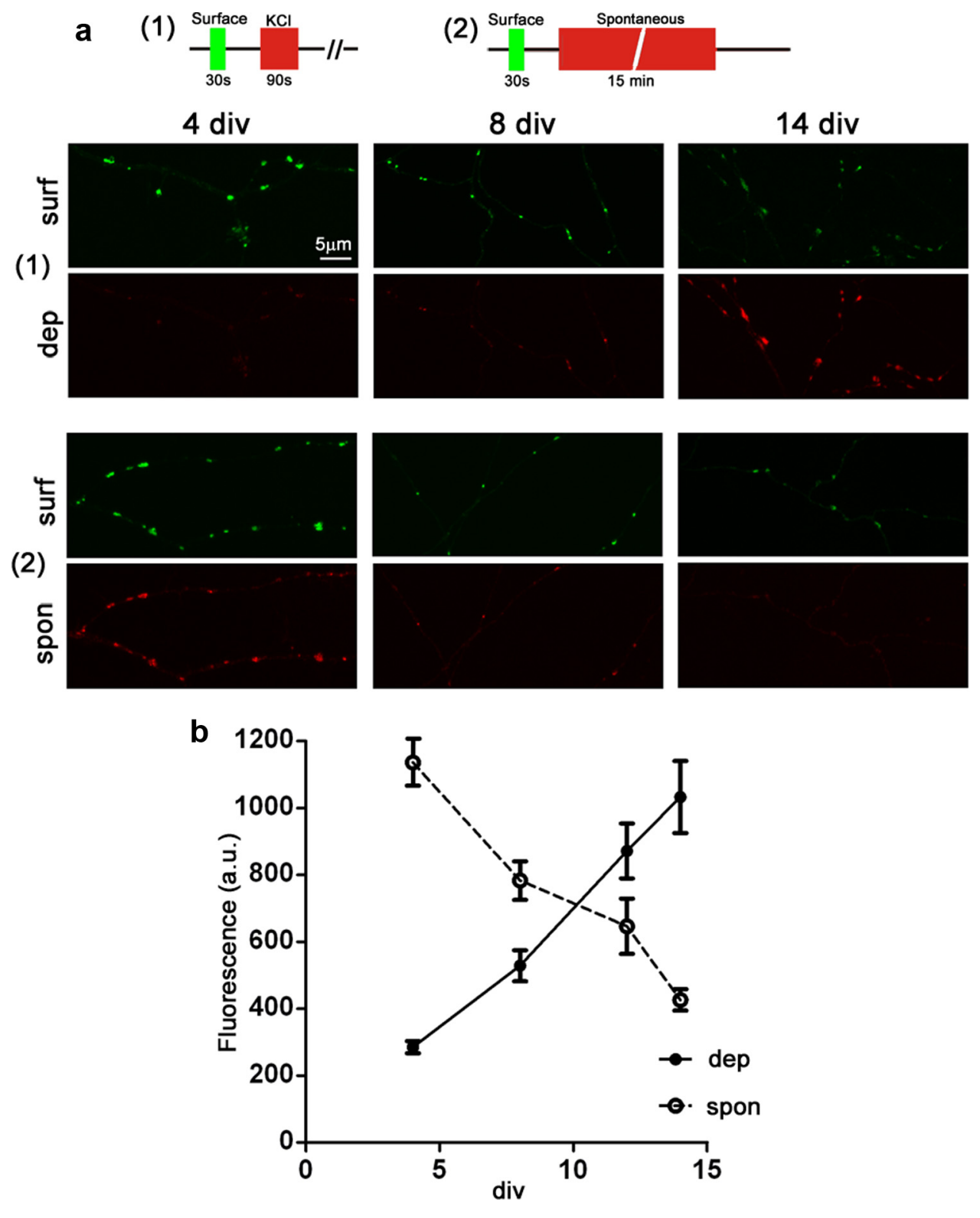

Figure 1. A developmental switch in vesicle release modes. $\boldsymbol{a}$, Schematic and fluorescence images: biosyn labeling of activity-dependent (dep) vesicle cycling (top, red) shows this progressively increases during development while spontaneous (spon) cycling (bottom, red) shows a corresponding decrease. Surface (surf) VAMP2 is labeled in green to identify release sites. $\boldsymbol{b}$, Plot of fluorescence intensities confirms this switch ( $n=9-19$ cells per condition/time point). Error bars represent SEM.

and Burrone, 2009). Bafilomycin A (Merck) was used at a final concentration of $1 \mu \mathrm{m}$. Neurons were depolarized with high $\mathrm{KCl}$ as above, or with 40 action potentials (APs) at $20 \mathrm{~Hz}[1 \mathrm{~ms} / 25 \mathrm{~mA}$ current pulses from parallel platinum electrodes $\sim 5 \mathrm{~mm}$ apart, using an SD9 stimulator (Grass Instruments)]. Images were obtained using an inverted microscope Olympus IX71 with a CCD camera (Coolsnap HQ; Photometrics) controlled by Slidebook software (Intelligent Imaging Innovations).

Labeling vesicles with cypHer-coupled synaptotagmin antibody. To label the evoked pool, neurons were incubated in $\alpha$ Syt1-cypHer (1:200; Synaptic Systems) in HBS plus 1\% BSA, $25 \mu \mathrm{m}$ APV, and $20 \mu \mathrm{m}$ CNQX and a train of 900 APs delivered at $20 \mathrm{~Hz}$. Neurons recovered for $5 \mathrm{~min}$ at $37^{\circ} \mathrm{C}$ (Hua et al., 2010). To label surface synaptotagmin molecules, neurons were incubated in $\alpha$ Syt1-cypHer as above for $1 \mathrm{~min}$ at room temperature. In either case, neurons were then washed before being transferred for imaging and further stimulation.

Data analysis. Images were analyzed using automized custom-written routines in MATLAB (MathWorks). For biosyn labeling, regions of interests (ROIs) of 4 pixels diameter were automatically picked based on surface staining (Figs. 1-3) and the average fluorescence intensity for each synapse calculated at its optimal focal point. For Figure 4, puncta were picked automatically based on evoked labeling, ROI size was increased to 6 pixels to accommodate adjacent PSD-95 staining, and inhibitory cells excluded.

To obtain pool separation index (PSI) values, we labeled either the evoked pool (D1) followed by the spontaneous pool (S2), or the spontaneous pool (S1) followed by the evoked pool (D2). We aimed to express the ratio of mean fluorescence intensity for a given loading method (either depolarization or spontaneous) when performed second to when performed first, so at first approximation, $\mathrm{PSI}_{\mathrm{dep}}=\mathrm{D} 2 / \mathrm{D} 1$ and $\mathrm{PSI}_{\mathrm{spon}}=$ S2/S1. Therefore, for a shared pool where the first stimulus is saturating, depletion of the pool by the first stimulus should result in no labeling by the second and hence a PSI value of zero. For independent pools, labeling should be approximately the same for both conditions and therefore the ratio should tend to 1 . The same fluorophore was used for each loading method, each in different neurons. However, where the evoked pool was labeled first, total fluorescence was contaminated by spontaneous cycling occurring during the $90 \mathrm{~s}$ depolarization. Therefore, to obtain the true value, we subtracted the mean fluorescence obtained during the $90 \mathrm{~s}$ 
a

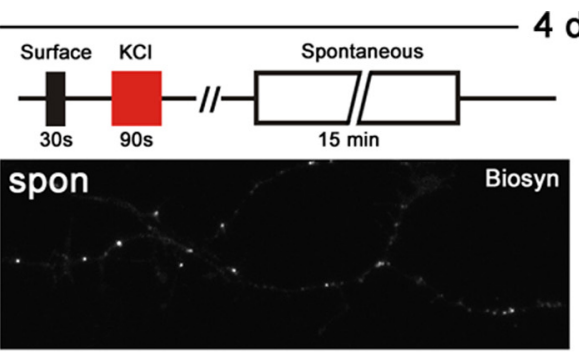

dep

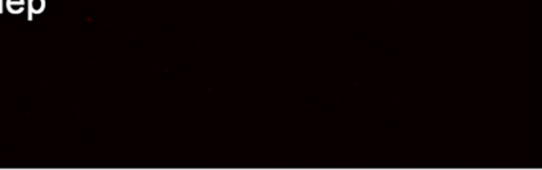

b

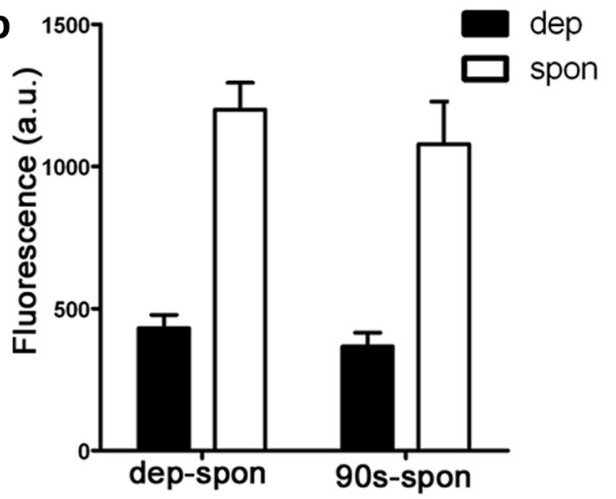

d
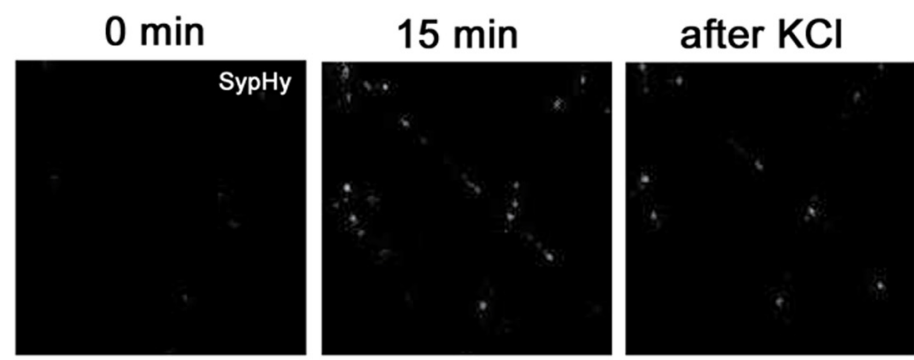

after $\mathrm{NH}_{4} \mathrm{Cl}$
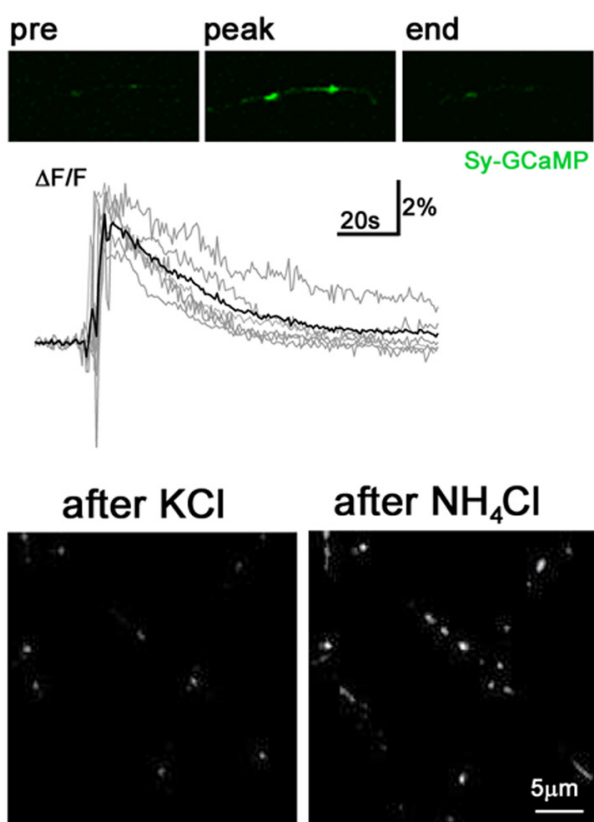

$\mathrm{NH}_{4} \mathrm{Cl}$

e

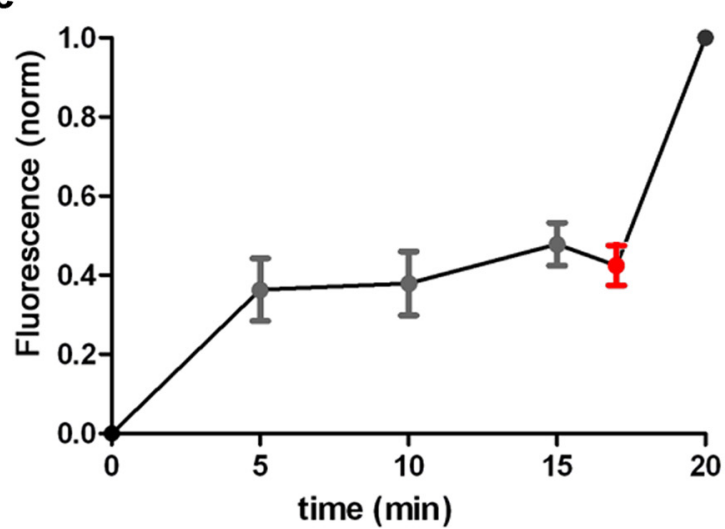

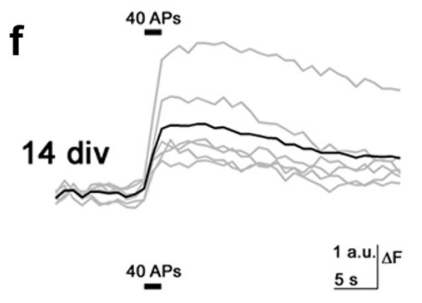

4 div

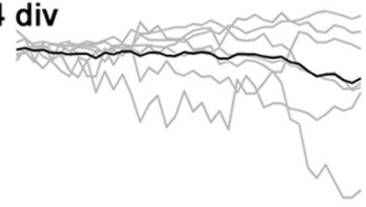

Figure 2. Evoked release is absent in young neurons. $\boldsymbol{a}, \boldsymbol{b}$, Schematic and fluorescence images (biosyn) to compare levels of cycling in $90 \mathrm{~s}$ at $4 \mathrm{DIV}$ with or without $60 \mathrm{~mm} \mathrm{KCl}$ reveal no differences between the two groups ( $n=10-15)$. c, Calcium transient in response to $\mathrm{KCl}$ in neurons expressing synaptophysin-GCaMP2 ( $n=6$; gray, individual traces; black, mean). $\boldsymbol{d}, \boldsymbol{e}$, Neurons expressing SypHy in Bafilomycin show no further increase in fluorescence following high KCl treatment $(n=5)$. Fluorescence intensities were normalized to total vesicle pool size as revealed by $\mathrm{NH}_{4} \mathrm{Cl}$ unquenching. $\boldsymbol{f}$, Stimulation with 40 action potentials at $20 \mathrm{~Hz}$ results in exocytotic response at 14 DIV ( $n=6$ ) but not at 4 DIV ( $n=7$ ). dep, activity-dependent; spon, spontaneous. Error bars represent SEM, $n$ represents number of cells. 
a

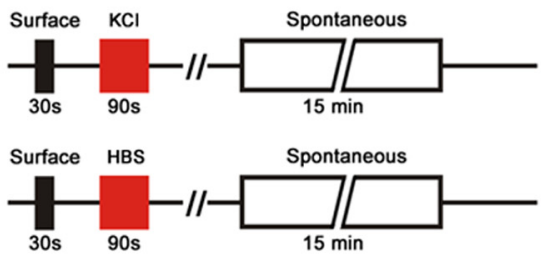

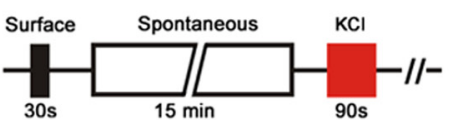

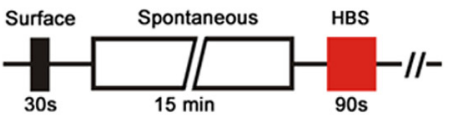

b

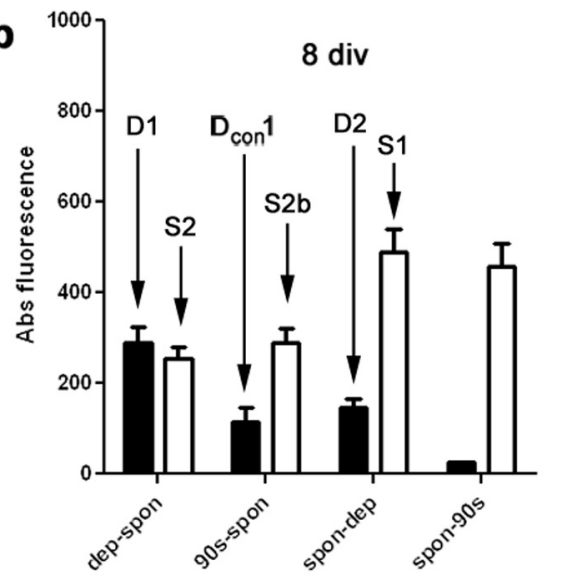

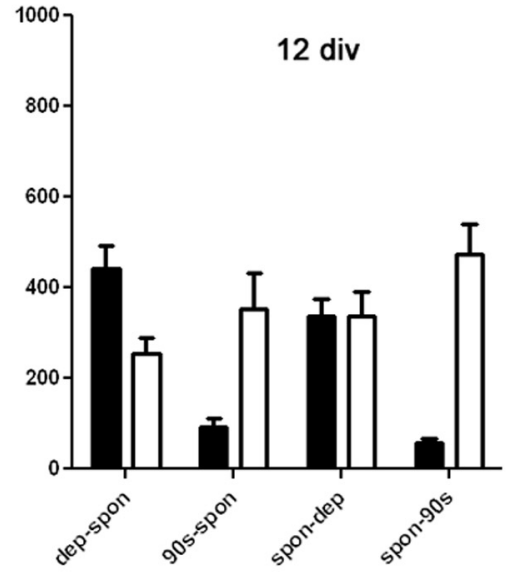

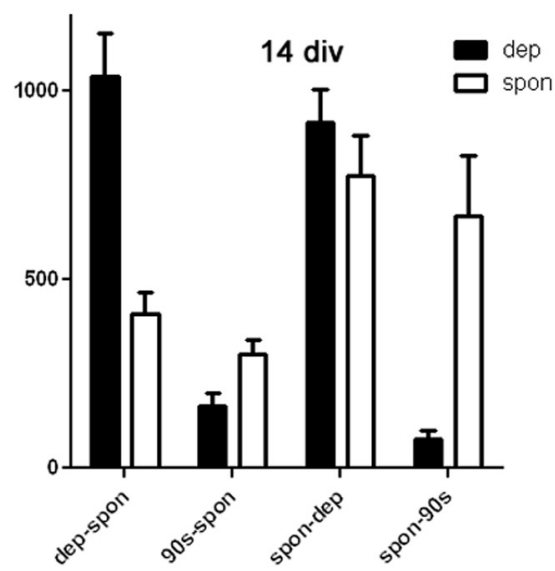

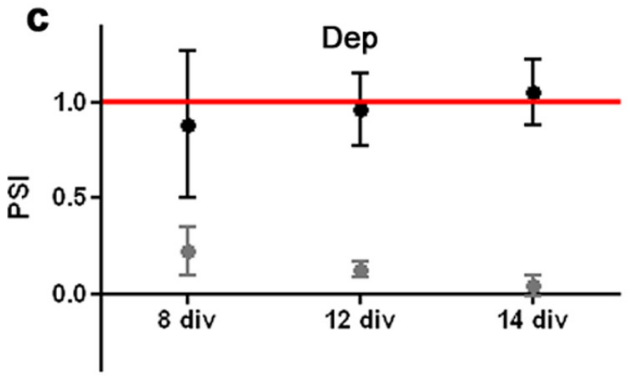
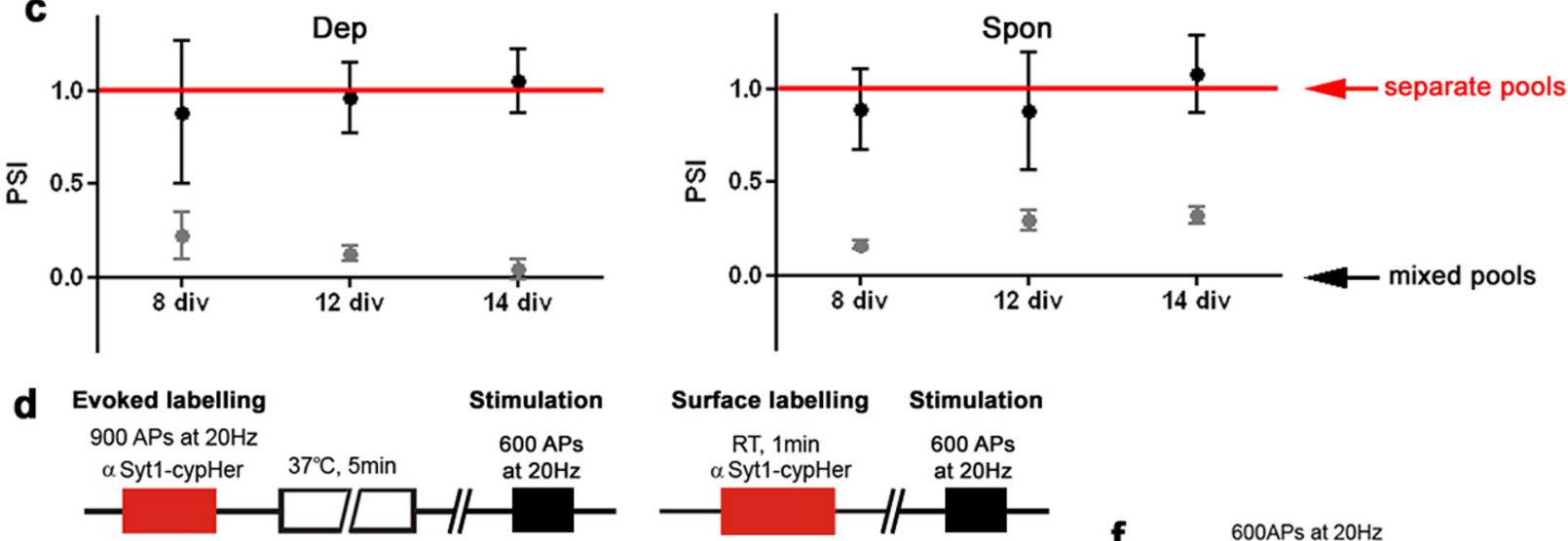

e
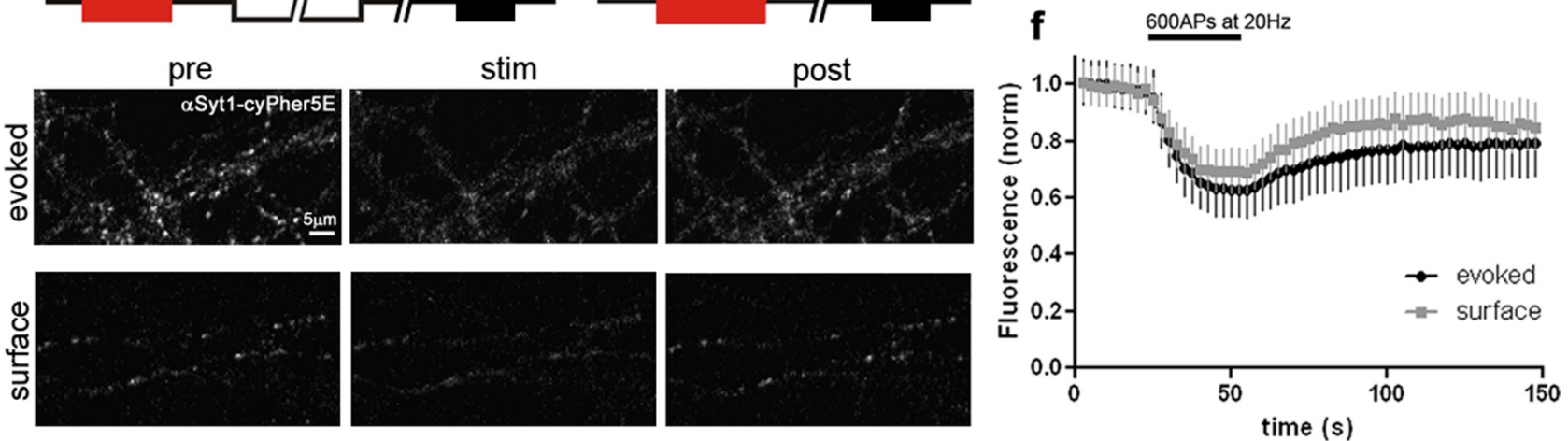

Figure 3. Evoked and spontaneous release derive from independent pools throughout development. $\boldsymbol{a}$, Schematic of protocol used to label sequential pools, controlling for the $90 \mathrm{~s}$ high $\mathrm{KCl}$ incubation in each case. $\boldsymbol{b}$, Graphs showing fluorescence intensities for the evoked (strep555) and spontaneous (strep647) vesicle pools at each developmental time point; labeling sequence indicated beneath columns, data used for PSI calculation indicated ( $n=24-37$ cells/time point). $c$, PSI plots for evoked (dep) and spontaneous (spon) pools. PSI values lie close to 1.0 ( $1=$ separate, $0=$ mixed) for all developmental time points. Controls (dep-dep or spon-spon) are shown in gray. $\boldsymbol{d}$, Schematic to show labeling of evoked and surface pools with $\alpha$ Syt1-cypHer and subsequent destaining with field stimulation of $600 \mathrm{APs}$ at $20 \mathrm{~Hz}$.e, Fluorescence images before, during, and after stimulation (stim) show quenching of labeled puncta during stimulation followed by recovery. $\boldsymbol{f}$, Mean fluorescence responses show very similar kinetics for both the evoked $(n=5)$ and surface $(n=5)$ pools. Error bars represent SEM.

control $\left(D_{\text {con }} 1\right)$, so that $\mathrm{PSI}_{\mathrm{dep}}=\mathrm{D} 2 /\left(\mathrm{D} 1-\mathrm{D}_{\mathrm{con}} 1\right)$. Where the spontaneous pool was labeled second, this value was artificially lower due to vesicles cycling spontaneously during the prior $90 \mathrm{~s}$ depolarization. Our experimental design did not include a measure of this in the same fluorophore as spontaneous labeling. Therefore, to calculate the amount of spontaneous labeling that occurs in $90 \mathrm{~s}$, we subtracted the spontaneous labeling obtained after the $90 \mathrm{~s}$ control (S2b) from the pooled data where spontaneous loading occurred first $\left(\mathrm{S}_{\text {pooled }}\right)$; then: $\mathrm{PSI}_{\text {spon }}=[\mathrm{S} 2+$ $\left.\left(\mathrm{S}_{\text {pooled }}-\mathrm{S} 2 \mathrm{~b}\right)\right] / \mathrm{S} 1$.

For controls, the same paradigm was performed twice. For spontaneous cycling: $\mathrm{PSI}_{\text {spon }}=\mathrm{S} 2 / \mathrm{S} 1$; for evoked cycling: $\mathrm{PSI}_{\mathrm{dep}}=\left(\mathrm{D} 2-\mathrm{D}_{\text {con }} 2\right) /$

$\left(\mathrm{D} 1-\mathrm{D}_{\mathrm{con}} 1\right)$. All data were expressed as mean $\pm \mathrm{SEM}$. 
a

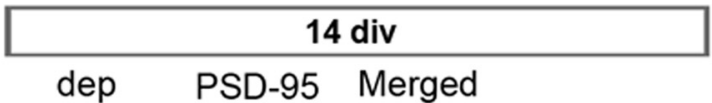

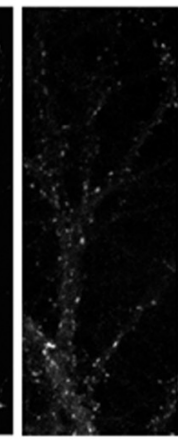
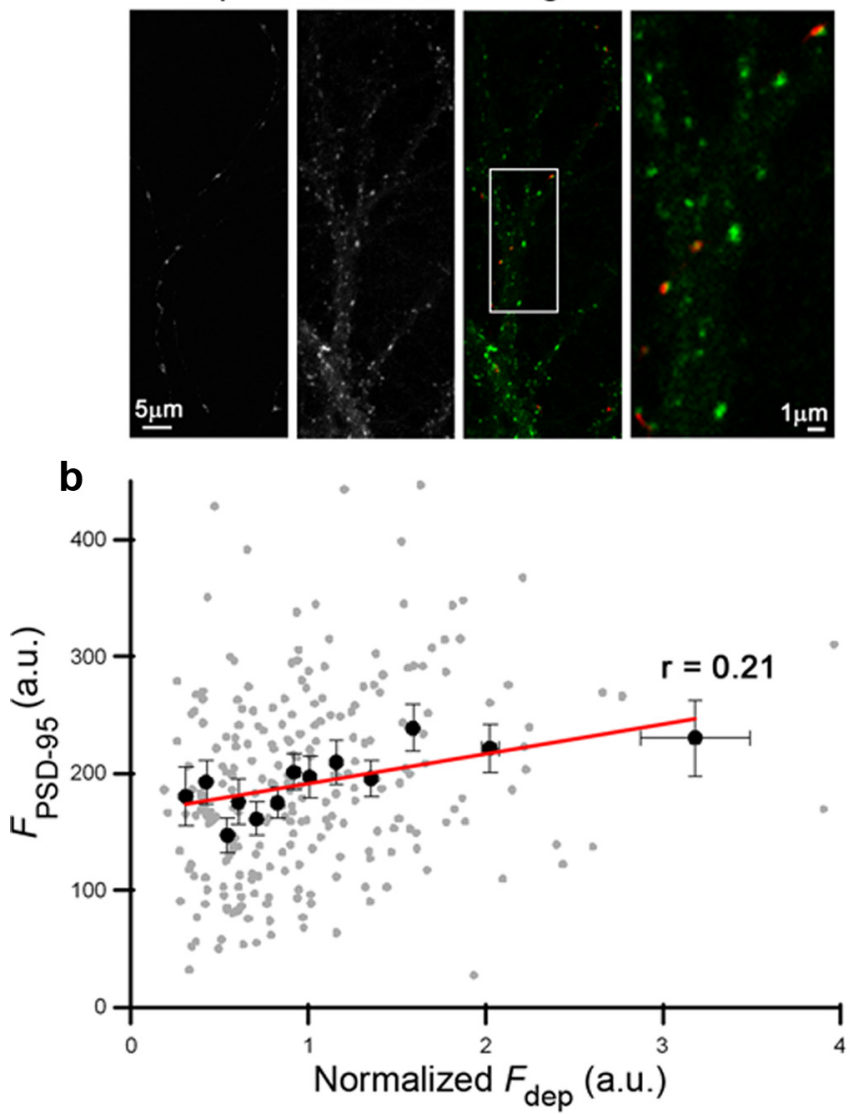

e

\section{7 div}
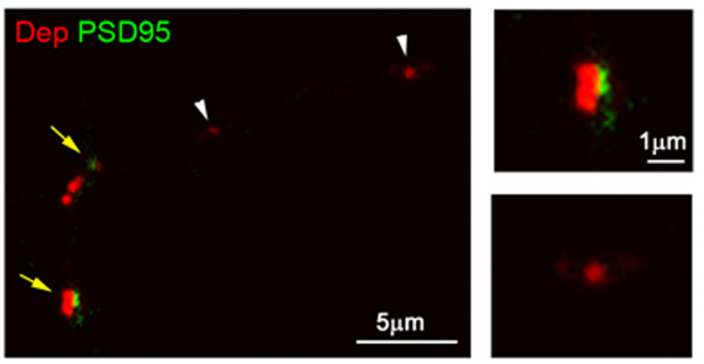

C
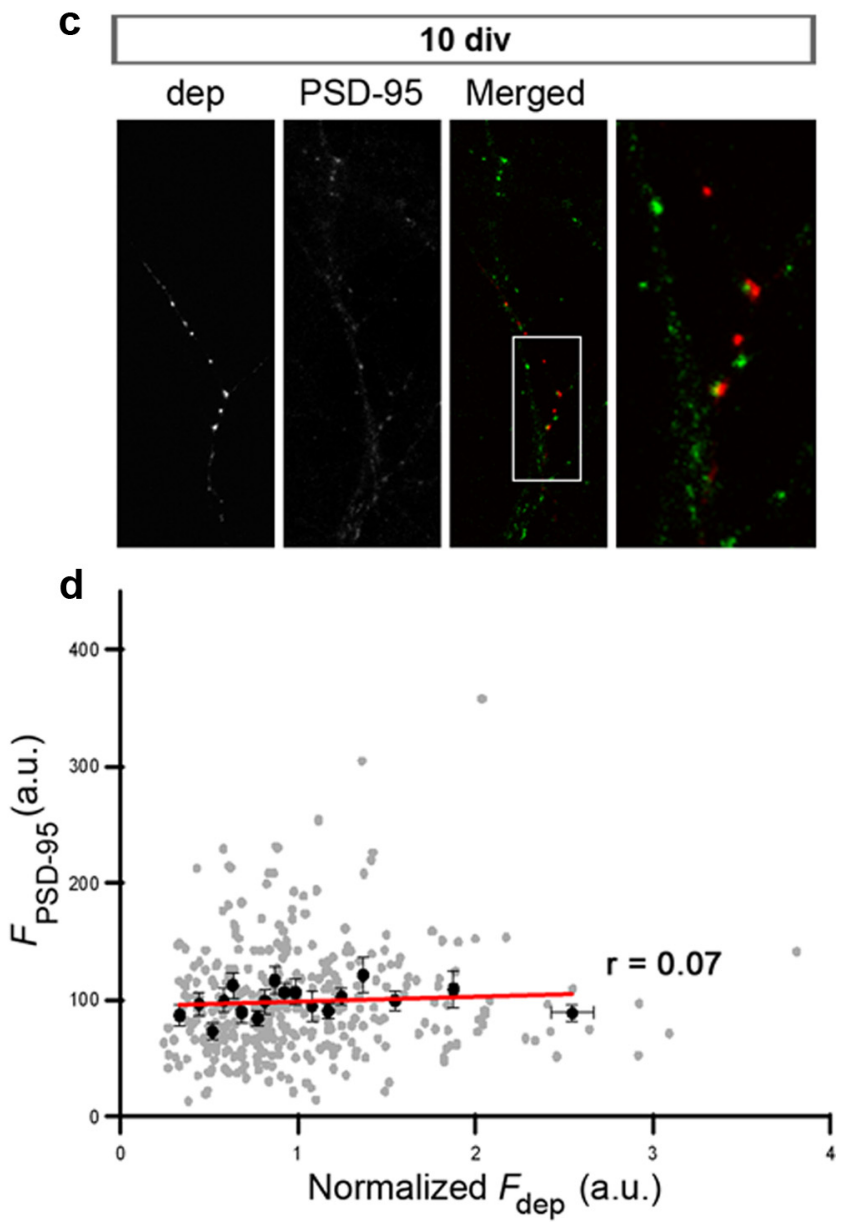

f

\section{6 div}

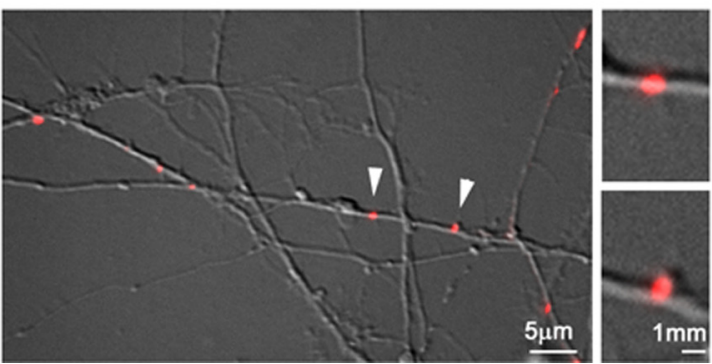

Figure 4. Induction of activity-dependent cycling is not dependent on postsynaptic structure or axodendritic contact. $\boldsymbol{a}$, Colabeling of the evoked pool (dep; red) and PSD-95 (green) at 14 DIV. Right, Enlargement of the boxed region showing colocalization of staining. $\boldsymbol{b}$, Plot of fluorescence intensity of PSD-95 staining as a function of the normalized fluorescence of the evoked pool $\left(F_{\text {dep }}\right.$; $n=244$ synapses, 11 cells). Individual synapses are in gray and the binned means (bin size $=10$ ) in black, best linear fit line (red) shows significant correlation. $\boldsymbol{c}, \boldsymbol{d}$, As in $\boldsymbol{a}$ and $\boldsymbol{b}$, respectively, but for 10 DIV, no correlation seen ( $n=475$ synapses, 12 cells). $\boldsymbol{e}$, At 7 DIV, while many sites of evoked release (red puncta) perfectly abut PSD-95 puncta (yellow arrows), others show no adjacent PSD-95 staining (white arrowheads), enlarged at right. $f$, At 6 DIV, many sites of evoked release (red puncta) do not have any nearby neuronal processes. Error bars represent SEM.

\section{Results}

A developmental switch in vesicle release modes

We used the biosyn probe, which consists of a luminally biotinylated VAMP2 (Fredj and Burrone, 2009), to quantify the size of vesicle pools that perform either evoked or spontaneous release in hippocampal neurons during development. Upon vesicle exocytosis, biosyn tightly binds to fluorescently labeled streptavidin to permanently tag vesicle pools. Importantly, the surface expression of VAMP2 was first quenched with another fluorescent streptavidin. Exocytosis was then triggered either by a $90 \mathrm{~s}$ incubation in $60 \mathrm{~mm}$ potassium chloride $(\mathrm{KCl})$ to depolarize neurons and label the evoked pool, or by a $15 \mathrm{~min}$ incubation at $37^{\circ} \mathrm{C}$ in TTX and $0 \mathrm{mM} \mathrm{Ca}^{2+}$ to label the spontaneous pool, at 4, 8, 12 and
14 DIV. We found that early in development, neurons display high levels of spontaneous labeling with low levels of activitydependent labeling; as maturation progresses, this switches over to a predominantly activity-dependent form of cycling (Fig. 1).

\section{Vesicular release is exclusively spontaneous early} in development

Given the high levels of spontaneous cycling in young neurons at 4 DIV, we hypothesized that the low levels of evoked staining observed may actually represent spontaneous release occurring over the $90 \mathrm{~s} \mathrm{KCl}$ incubation. Indeed, we found identical levels of staining when comparing neurons challenged for $90 \mathrm{~s}$ with streptavidin-Alexa555 either in the presence or absence of $60 \mathrm{~mm}$ 
$\mathrm{KCl}$ (Fig. 2a,b), despite the induction of robust, local calcium transients at the presynaptic terminal by high $\mathrm{KCl}$ (Fig. $2 c$ ), indicating the absence of activity-dependent cycling in axons at 4 DIV. Since contradictory data have been described previously (Kraszewski et al., 1995; Coco et al., 1998; Sabo and McAllister, 2003) and to further confirm our findings, we also used the $\mathrm{pH}-$ sensitive reporter of synaptic vesicle cycling, synaptophysinpHluorin (SypHy) to measure the levels of evoked and spontaneous release in 4 DIV neurons. SypHy-expressing neurons were incubated in the $\mathrm{v}$-ATPase inhibitor bafilomycin to prevent reacidification of vesicles following exocytosis, thus providing a cumulative measure of release. Saturation of spontaneous release was seen within a $15 \mathrm{~min}$ incubation period, but no further increases were seen upon addition of $60 \mathrm{~mm} \mathrm{KCl}$ (Fig. $2 d, e)$. Finally, in a separate set of experiments, we stimulated SypHy-expressing cells with a brief train of 40 action potentials at $20 \mathrm{~Hz}$ (without bafilomycin), which evoked exocytosis at $14 \mathrm{DIV}$ but no response at 4 DIV (Fig. $2 f$ ). Together, this is compelling evidence that release is exclusively spontaneous early in development.

\section{Evoked and spontaneous release derive from independent pools throughout development}

Next, we investigated whether these two modes of release derive from a communal pool or were segregated during development. Sequential labeling of pools with spectrally distinct streptavidin molecules indicates the extent to which vesicles derive from a communal pool, as vesicles tagged following one mode of release will deplete the vesicles available for subsequent labeling. However, if the pools are independent, then the order of labeling will not affect pool size. Four parallel experiments were conducted (Fig. $3 a$ ) to label the evoked pool followed by the spontaneous pool and vice versa, including $90 \mathrm{~s}$ controls for the high $\mathrm{KCl}$ treatments. The results are shown in Figure $3 b$. We calculated a pool separation index (PSI) (see Materials and Methods, above) for both the evoked and spontaneous pools with a range of 0 (complete mixing) to 1 (complete separation). We found that for both evoked and spontaneous labeling, the PSI values at all stages studied lay close to 1 (Fig. 3c), while controls where the same pool was sequentially labeled (Fig. $3 c$, gray) were much closer to 0 . Together, these results indicate that spontaneous and activitydependent release originates from independent vesicle pools throughout development.

These results contradict recent data in mature synapses using a $\mathrm{pH}$-sensitive reporter cypHer5E coupled to an antibody against the luminal domain of synaptotagmin 1 ( $\alpha$ Syt1-cypHer), which was proposed to allow imaging of vesicles after they are rereleased (Hua et al., 2010). Hua et al.'s (2010) findings showed that regardless of loading method (activity or spontaneous), $\alpha$ Syt1cypHer-labeled vesicles that were subsequently mobilized in an activity-dependent manner showed similar release kinetics, suggesting that vesicles are mobilized from the same pool for both release modes. However, this study did not address the possible contribution of the large amount of synaptotagmin1 (Syt1) already present on the surface of the presynaptic membrane that also undergoes vesicle recycling. We therefore used $\alpha$ Syt1cypHer to label either the evoked pool (with 600 action potentials at $20 \mathrm{~Hz}$ ) or the surface population of Syt 1 only (1 min incubation at room temperature) (Fig. $3 d$ ), and then stimulated both groups of neurons with 600 action potentials at $20 \mathrm{~Hz}$ to follow their rerelease. We found almost identical decay and recovery curves for both conditions (Fig. 3e,f), indicating that surface Syt 1 mixes extensively with recycling vesicles, as for other synaptic vesicle proteins (Fernández-Alfonso et al., 2006; Wienisch and Klingauf, 2006), and will therefore erroneously report activitydependent cycling, even when spontaneous vesicles are labeled. We conclude that this method is not suitable for investigating pool segregation.

\section{Cell-autonomous switch in release modes}

Finally, our results indicate that evoked release in these neurons is absent early in development. As the switch from spontaneous to evoked release correlates temporally with the peak of synapse formation, we hypothesized that the initiation of activitydependent cycling might be dependent on postsynaptic contact. To label the evoked pool without contamination from spontaneously cycling vesicles, neurons from 10 and 14 DIV underwent labeling for spontaneous cycling followed by depolarization with $60 \mathrm{~mm} \mathrm{KCl}$ and were then fixed and processed for PSD-95 immunocytochemistry. Puncta were selected automatically and axons with no adjacent PSD-95 puncta (presumably inhibitory neurons) were excluded. Although we found a significant correlation between evoked labeling and PSD-95 staining at 14 DIV $(r=$ $0.21, p<0.0001$; Fig. $4 a, b)$, there was no correlation at $10 \mathrm{DIV}$ $(r=0.07, p=0.18$; Fig. $4 c, d)$. Furthermore, at 7 DIV, we found many sites of evoked release with no adjacent PSD-95 staining (Fig. 4e). This suggests that the postsynaptic density is not responsible for the progressive increase in activity-dependent release. We then examined whether the presence of axodendritic contact was important by using brightfield Nomarski optics to view all neuronal processes at 6 DIV. We found a significant number of activity-dependent vesicles with no other nearby process (Fig. 4f), indicating that stable axodendritic contact is not necessary for the commencement of evoked release. We propose that the initiation of activity-dependent cycling is likely to be set autonomously on the presynaptic side.

\section{Discussion}

We have shown that during development of hippocampal neurons, release modes change dramatically: from exclusively high levels of spontaneous cycling in young neurons to the mature phenotype of strong evoked release and low-level spontaneous release. This switch occurred in a graded manner, with a progressive downregulation of spontaneous cycling and a corresponding increase in activity-dependent cycling. Moreover, the appearance of activity-dependent release was shown to be an autonomous presynaptic event, not dependent on axodendritic contact. Furthermore, our results confirm that the spontaneous and evoked modes of release draw from separate pools of vesicles in mature neurons and further extend this observation to show that the segregation of vesicle pools occurs throughout neuronal development.

We used the biosyn technique to perform a detailed quantitative assessment of spontaneous and activity-dependent cycling throughout development. Our methods show that early in development, before axodendritic contact is established, there is a large pool of vesicles that recycles constitutively. The size of this pool is dramatically reduced during axonal maturation, providing important evidence for a developmental program controlling spontaneous neurotransmitter release. The progressive increase in evoked pool size during development that we see with biosyn is in agreement with a previous study using FM2-10 to label recycling vesicles (Mozhayeva et al., 2002). While the physiological function of spontaneous release remains unclear, our findings will now force us to extend our search for function to much earlier developmental time points. 
A surprising result in our data was the absence of activitydependent cycling early in development (4 DIV). Many previous studies have used high $\mathrm{KCl}$ to attempt to label vesicles with either styryl dyes or Syt1 antibodies during neuronal development (Kraszewski et al., 1995; Dai and Peng, 1996; Coco et al., 1998; Zakharenko et al., 1999; Sabo and McAllister, 2003; Tojima et al., 2007). Although it is possible that in some studies, the long exposures to $\mathrm{KCl}$ may inadvertently result in labeling of the highly active spontaneous pool, others have clearly shown activitydependent vesicle cycling in young neurons (Dai and Peng, 1996; Zakharenko et al., 1999; Tojima et al., 2007). However, these studies have been performed in different species and culture systems, as well as distinct neuronal types: chick dorsal root ganglion cells, cultured Xenopus spinal neurons, and dissociated rodent hippocampal neurons. It is possible that species or cell-type differences could account for this discrepancy.

Earlier studies investigating pool identity used variants of the styryl FM dyes with conflicting results (Sara et al., 2005; Groemer and Klingauf, 2007; Atasoy et al., 2008). To bypass some of the inherent technical problems of using these dyes, we previously used the biosyn technique to permanently label vesicle pools and demonstrated that spontaneous and evoked release derive from separate pools (Fredj and Burrone, 2009), but this view has recently been challenged (Hua et al., 2010; Wilhelm et al., 2010). However, technical issues with the techniques used suggest that there may be inherent problems with the methods of studying vesicle cycling.

In one paper (Wilhelm et al., 2010), most of the experiments use FM dyes, with issues that have been discussed previously (Fredj and Burrone, 2009). In a key experiment, Wilhelm et al. (2010) use the $\mathrm{pH}$-sensitive exocytosis reporter, synaptopHluorin, to demonstrate similar levels of exocytosis due to both active and spontaneous cycling following labeling of the activitydependent pool. However, the authors removed the protonpump inhibitor folimycin from the medium so that, over the 15 min period used to show exocytosis of the evoked pool by spontaneous release, reacidification might occur due to potential reversibility of these drugs (Sankaranarayanan and Ryan, 2001; Chang and Mennerick, 2010), which could account for the observed change in fluorescence. More compellingly, in Hua et al. (2010), the authors used two similar techniques: a modified biosyn probe coupled to a $\mathrm{pH}$-sensitive reporter cypHer5E, and a cypHer5E-coupled antibody against the luminal domain of Syt1 ( $\alpha$ Syt1-cypHer). These appear to show that after loading vesicles spontaneously or in response to activity, rerelease follows similar destaining curves with electrical stimulation. However, in both cases, surface expression of either VAMP2 or Syt1 was not accounted for. VAMP2 and Syt1 proteins are highly expressed on the synaptic plasma membrane (Fernández-Alfonso et al., 2006; Wienisch and Klingauf, 2006; Fredj and Burrone, 2009). Normalization of the data means that any contamination from surface molecules to the recycling pool would result in similar but artifactual destaining curves. We used $\alpha$ Syt1-cypHer to demonstrate that, by solely labeling surface Syt 1 molecules, an almost identical destaining curve is observed in response to electrical stimulation to that found after labeling the activity-dependent pool. This confirms previous data indicating extensive mixing of surface Syt1 with the recycling pool (Fernández-Alfonso et al., 2006; Wienisch and Klingauf, 2006) and renders it impossible to draw conclusions regarding pool origin from these experiments.

The origin of spontaneous and activity-dependent vesicle pools has not been studied previously. Here, we demonstrate that these two pools are effectively independent from the beginning of development and throughout the process of maturation. Recently, an increasing body of evidence has indicated that the spontaneous release of neurotransmitter from mature synaptic terminals, in the form of miniature excitatory postsynaptic currents (mEPSCs), can play critical roles in synaptic function. Not only can mEPSCs influence neuronal firing (Carter and Regehr, 2002; Sharma and Vijayaraghavan, 2003), but they have also been shown to regulate synaptic scaling via effects on local dendritic protein synthesis (Sutton et al., 2006). While we do not yet understand the role of spontaneous release before axodendritic contact and how this might relate to the mature state, it is possible that the graded switch in types of release might reflect an underlying switch in function.

Since activity-dependent cycling is not present at early time points, but is initialized later in development during the period of synapse formation, we revisited the issue of whether the postsynaptic compartment is responsible for its induction. Our results indicate that contact with a postsynaptic dendrite is not necessary for the appearance of activity-dependent cycling. Furthermore, the correlation between the size of the activity-dependent pool and PSD-95 staining at individual synapses was significant at 14 DIV but not at 10 DIV. Previous data has shown matching of synaptic strength between presynaptic and postsynaptic compartments, both structurally (Schikorski and Stevens, 1999) and functionally (Tokuoka and Goda, 2008; Kay et al., 2011) in mature neurons. Previous data from our lab has demonstrated that, at 10 DIV, individual synapses show strong correlation at a structural level but no correlation functionally (Kay et al., 2011), begging the question: which side drives functional matching? Since we now show that a functional presynaptic readout fails to correlate with a structural postsynaptic readout at this developmental stage, it is likely that changes in presynaptic function are responsible for the emergence of a functional correlation. Based on the dramatic developmental redistribution of presynaptic vesicle pools described here, this possibility becomes an even more attractive option.

\section{References}

Atasoy D, Ertunc M, Moulder KL, Blackwell J, Chung C, Su J, Kavalali ET (2008) Spontaneous and evoked glutamate release activates two populations of NMDA receptors with limited overlap. J Neurosci 28:10151-10166

Blue ME, Parnavelas JG (1983) The formation and maturation of synapses in the visual cortex of the rat. II. Quantitative analysis. J Neurocytol 12:697-712.

Carter AG, Regehr WG (2002) Quantal events shape cerebellar interneuron firing. Nat Neurosci 5:1309-1318.

Chang CY, Mennerick S (2010) Dynamic modulation of phasic and asynchronous glutamate release in hippocampal synapses. J Neurophysiol 103:392-401.

Chung C, Barylko B, Leitz J, Liu X, Kavalali ET (2010) Acute dynamin inhibition dissects synaptic vesicle recycling pathways that drive spontaneous and evoked neurotransmission. J Neurosci 30:1363-1376.

Coco S, Verderio C, De Camilli P, Matteoli M (1998) Calcium dependence of synaptic vesicle recycling before and after synaptogenesis. J Neurochem 71:1987-1992.

Dai Z, Peng HB (1996) Dynamics of synaptic vesicles in cultured spinal cord neurons in relationship to synaptogenesis. Mol Cell Neurosci 7:443-452.

Fernández-Alfonso T, Kwan R, Ryan TA (2006) Synaptic vesicles interchange their membrane proteins with a large surface reservoir during recycling. Neuron 51:179-186.

Fredj NB, Burrone J (2009) A resting pool of vesicles is responsible for spontaneous vesicle fusion at the synapse. Nat Neurosci 12:751-758.

Groemer TW, Klingauf J (2007) Synaptic vesicles recycling spontaneously and during activity belong to the same vesicle pool. Nat Neurosci 10:145-147.

Hua Y, Sinha R, Martineau M, Kahms M, Klingauf J (2010) A common 
origin of synaptic vesicles undergoing evoked and spontaneous fusion. Nat Neurosci 13:1451-1453.

Kay L, Humphreys L, Eickholt BJ, Burrone J (2011) Neuronal activity drives matching of pre- and postsynaptic function during synapse maturation. Nat Neurosci 14:688-690.

Kraszewski K, Mundigl O, Daniell L, Verderio C, Matteoli M, De Camilli P (1995) Synaptic vesicle dynamics in living cultured hippocampal neurons visualized with CY3-conjugated antibodies directed against the lumenal domain of synaptotagmin. J Neurosci 15:4328-4342.

Matteoli M, Takei K, Perin MS, Südhof TC, De Camilli P (1992) Exoendocytotic recycling of synaptic vesicles in developing processes of cultured hippocampal neurons. J Cell Biol 117:849-861.

Mozhayeva MG, Sara Y, Liu X, Kavalali ET (2002) Development of vesicle pools during maturation of hippocampal synapses. J Neurosci 22:654-665.

Sabo SL, McAllister AK (2003) Mobility and cycling of synaptic proteincontaining vesicles in axonal growth cone filopodia. Nat Neurosci $6: 1264-1269$.

Sankaranarayanan S, Ryan TA (2001) Calcium accelerates endocytosis of vSNAREs at hippocampal synapses. Nat Neurosci 4:129-136.

Sara Y, Virmani T, Deák F, Liu X, Kavalali ET (2005) An isolated pool of vesicles recycles at rest and drives spontaneous neurotransmission. Neuron 45:563-573.
Schikorski T, Stevens CF (1999) Quantitative fine-structural analysis of olfactory cortical synapses. Proc Natl Acad Sci U S A 96:4107-4112.

Sharma G, Vijayaraghavan S (2003) Modulation of presynaptic store calcium induces release of glutamate and postsynaptic firing. Neuron 38:929-939.

Sutton MA, Ito HT, Cressy P, Kempf C, Woo JC, Schuman EM (2006) Miniature neurotransmission stabilizes synaptic function via tonic suppression of local dendritic protein synthesis. Cell 125:785-799.

Tojima T, Akiyama H, Itofusa R, Li Y, Katayama H, Miyawaki A, Kamiguchi H (2007) Attractive axon guidance involves asymmetric membrane transport and exocytosis in the growth cone. Nat Neurosci 10:58-66.

Tokuoka H, Goda Y (2008) Activity-dependent coordination of presynaptic release probability and postsynaptic GluR2 abundance at single synapses. Proc Natl Acad Sci U S A 105:14656-14661.

Wienisch M, Klingauf J (2006) Vesicular proteins exocytosed and subsequently retrieved by compensatory endocytosis are nonidentical. Nat Neurosci 9:1019-1027.

Wilhelm BG, Groemer TW, Rizzoli SO (2010) The same synaptic vesicles drive active and spontaneous release. Nat Neurosci 13:1454-1456.

Zakharenko S, Chang S, O’Donoghue M, Popov SV (1999) Neurotransmitter secretion along growing nerve processes: comparison with synaptic vesicle exocytosis. J Cell Biol 144:507-518. 